\title{
Qualidade de vida de pacientes renais crônicos em tratamento dialítico hemodialítico em Guaraí- TO
}

\section{Quality of life of chronic renal patients undergoing hemodialysis treatment in Guaraí- TO}

Kamilla Sobral Rodrigues ${ }^{1}$, Marly Ribeiro Dos Santos², Adriana Keila Dias ${ }^{3}$, Giullia Bianca Ferraciolli do Couto $^{4}$, Reobbe Aguiar Pereira ${ }^{5}$, Glaucya Wanderley Santos Markus ${ }^{6}$

\section{RESUMO}

A Insuficiência Renal Crônica (IRC) é uma patologia de graduação lenta, caracterizada mediante alterações estruturais e/ou funcionais do sistema renal, órgão vital na manutenção do equilíbrio hidroeletrolítico. Objetivo do estudo em avaliar a qualidade de vida de pacientes renais crônicos em terapia substitutiva que realizam a Hemodiálise e Diálise Peritoneal. Pesquisa de campo de abordagem qualitativa exploratóriodescritivo, realizada na Clínica de Educação para a Saúde (CEPS) do Instituto Educacional Santa Catarina - IESC/FAG no município de Guaraí - TO. A população desse estudo é composta por 07 pacientes portadores de IRC em Terapia Renal Substitutiva (TRS). Foi aplicado o questionário WHOQOL para avaliação da Qualidade de Vida (QV), as informações coletadas foram organizadas e apresentadas em forma de gráficos. No domínio QV, $71,4 \%$ dos participantes da pesquisa declaram ter uma QV de vida ruim ou muito ruim e apenas $28,6 \%$ afirmam boa $Q V$, o que resulta o quanto a doença interfere nos aspectos biopsicossociais do indivíduo. A prevalência de pacientes em Hemodiálise é significativamente maior se comparados à Diálise Peritoneal. A QV dos pacientes em TRS é substancialmente menor em função da sobrecarga causada pela doença o que reforça a necessidade de medidas de prevenção.

Palavras-chave: Modalidades terapêuticas; Insuficiência; Prevenção.

\section{ABSTRACT}

Chronic Renal Insufficiency (CRF) is a slow-graded pathology, characterized by structural and / or functional changes in renal function, a vital organ in maintaining hydroelectrolytic balance. To evaluate the quality of life of chronic renal patients undergoing substitution therapy undergoing Hemodialysis and Peritoneal Dialysis. Field research with a qualitative exploratorydescriptive approach, carried out at the Health Education Clinic (CEPS) of the Instituto Educacional Santa Catarina - IESC / FAG in the city of Guaraí - TO. The study population consists of 07 patients with CRF undergoing Renal Replacement Therapy (RRT). The WHOQOL questionnaire was applied to assess Quality of Life (QOL), the information collected was organized and presented in the form of graphs. In the QOL domain, $71.4 \%$ of the survey participants declared their QOL to be poor or very bad and only $28.6 \%$ reported good QOL, these results show how much the disease interferes with the individual's biopsychosocial aspects. The prevalence of patients on Hemodialysis is significantly higher when compared to Peritoneal Dialysis. The QoL of patients on RRT is substantially lower due to the burden caused by the disease, which reinforces the need for preventive measures.
${ }^{1}$ Graduada em Enfermagem na Faculdade Guaraí-FAG. E-mail: kamila.sobralrodrigues@gmail.co m

${ }^{2}$ Graduada em Enfermagem na Faculdade Guaraí-FAG. E-mail: marliribeiro_santos@hotmail.co m

${ }^{3}$ Enfermeira. Mestra em Ciências Ambientais. Doutoranda em Engenharia Biomédica. Docente da Faculdade Guaraí-FAG. E-mail: adrianakeiladias@hotmail.com

${ }^{4}$ Enfermeira. Mestra em Bioengenharia com Ênfase em Saúde. Docente da Faculdade Guaraí-FAG. giulliabianca@hotmail.com

${ }^{5}$ Enfermeiro. Mestre em Ciências Ambientais. Doutorando em Engenharia Biomédica. E-mail: enfreobbe@gmail.com

${ }^{6}$ Enfermeira. Mestra E-mail: Bioengenharia com Ênfase em Saúde. Docente da Faculdade Guaraí-FAG. E-mail: glaucyamarkus@outlook.com

Keywords: Therapeutic modalities; Insufficiency; Prevention. 


\section{INTRODUÇÁOO}

As doenças crônicas vêm se destacando mundialmente como um problema de saúde pública que exigem mudanças comportamentais para adequação de um novo estilo de vida, a qual se destaca a Insuficiência Renal Crônica (IRC), patologia de graduação lenta, caracterizada mediante alterações estruturais e/ou funcionais do sistema renal, o que ocasiona diminuição da taxa de filtração dos glomérulos, decorrente de vários fatores seja eles genéticos, fisiológicos, ou por excesso de produtos hidrogenados (ureia e creatinina) no sangue, que impossibilitam os rins de manter a homeostase do organismo, gerando impacto negativo na qualidade de vida dos indivíduos. ${ }^{1}$

Os renais crônicos passam por uma mudança radical provocada pelo tratamento, podendo ser considerado fonte de estresse, baixa autoestima e necessidade de adaptação a nova rotina, visto que a mesma faz-se essencial para manutenção da saúde, seja ele feito através de Diálise peritoneal ou Hemodiálise, na qual exige do organismo muita energia para realização das seções dialisadoras. De modo geral, o tratamento dialítico acarreta alterações fisiológicas no organismo, como por exemplo diabetes mellitus, hipertensão arterial e glomerulonefrites. ${ }^{2,19}$

A Sociedade Brasileira de Nefrologia (SBN) relata que a incidência e prevalência de IRC tem aumentado de forma progressiva nos últimos anos, visto que em países desenvolvidos há uma estimativa de $10 \%$ a 13\%, gerando altos custos no tratamento, principalmente à pacientes em uso de terapia renal substitutiva (TRS) que vêm aumentando gradativamente em todo o mundo, inclusive no Brasil com estimativa de 100.397 pessoas dialisadas em $2018 .^{1}$

Os portadores de Insuficiência Renal dependentes de Terapias Renais Substitutivas (Hemodiálise e Diálise peritoneal) sofrem inúmeras limitações nos aspectos biopsicossociais, que refletem em insegurança quanto a realização de suas atividades de vida diária, sentimento de não aceitação a realidade que o tratamento impõem, limitações físicas, sexuais, psicológicas, familiares e sociais, que afetam a qualidade de vida, dispondo de sentimento negativos, medo do prognóstico, incapacidade, dependência econômica e alteração da autoimagem, posto isso, os pacientes devem valer-se de estratégias de enfrentamento para lidar com a negatividade, dispondo do apoio familiar e da resiliência. ${ }^{3}$

A qualidade de vida (QV) é caracterizada por fatores multidimensionais que abrangem os aspectos biopsicossociais, dando ênfase ao bem-estar do indivíduo, desta 
forma, para uma avaliação precisa, faz-se necessário a utilização de instrumentos psicométrico com parâmetros fidedignos como o WHOQOL, instrumento produzido pela Organização Mundial de Saúde (OMS), que permite melhor avaliação individual da QV em grupos distintos. ${ }^{4}$ Assim, este tem como objetivo geral avaliar a qualidade de vida de pacientes renais crônicos em terapia substitutiva que realizam a Hemodiálise e a Diálise Peritoneal, bem como avaliar as condições clínicas e psicossociais mediante as terapias indicadas.

\section{MATERIAL E METODOS}

Trata-se de um estudo com abordagem qualitativa, exploratório e descritivo. O prosseguimento do estudo atendeu às normas nacionais e internacionais de ética em pesquisa envolvendo seres humanos, resguardados pela a Resolução № 466, de 12 de 2012 do Conselho Nacional de Saúde (CNS). A pesquisa obteve a aprovação do Comitê de Ética em Pesquisa da Universidade Estadual do Tocantins - UNITINS, campus de PalmasTO, emitido sob parecer № 4.314.963.

A população deste estudo foi composta por 07 pacientes portadores de IRC em tratamento por meio de diálise peritoneal e hemodiálise. Foram selecionados pacientes que apresentam doença renal crônica, com no mínimo 90 dias de tratamento, maiores de idade, em condições clínicas estáveis e adequada para responder aos questionários. Vale ressaltar que os pacientes foram informados sobre os objetivos da pesquisa e a confidencialidade dos dados. Após concordarem em participar do estudo, assinaram o Termo de Consentimento Livre e Esclarecido (TCLE). Foram excluídos da pesquisa os pacientes impossibilitados de responder as questões apresentadas por déficit de autoconhecimento e aqueles que se abstiveram em assinar o TCLE.

A pesquisa foi realizada na Clínica de Educação para a Saúde (CEPS), do Instituto Educacional Santa Catarina- IESC/FAG, no município do Guaraí- Tocantins. A seleção dos participantes da pesquisa foi mediante o mesmo ser portador de insuficiência renal crônica e realizando tratamento de hemodiálise dialíticos. Os pacientes foram esclarecidos sobre as finalidades e os objetivos da pesquisa e sobre a interrupção da mesma instantaneamente, caso algum sentisse indícios de incomodidade e/ou desejasse desistir de participar do estudo.

A coleta de dados foi realizada após aprovação do comitê de ética, através do questionário WHOQOL, preenchido pela as pesquisadoras responsáveis. No mesmo, havia 
um total de 13 perguntas, sendo todas objetivas. O questionário WHOQOL trata-se da qualidade de vida nos âmbitos biopsicossocial dos portadores de doença renal em tratamento dialítico, sendo que as questões oferecem alternativas que se adequa ao perfil de cada indivíduo.

As informações coletadas foram reguladas, organizadas e sucessivamente agrupadas e tabuladas no programa Microsoft Excel, fazendo uso do cálculo de porcentagem simples, apresentadas em formas gráficos. Em seguida, os resultados foram discutidos através de referencial teórico em revisões bibliográficas.

\section{RESULTADOS E DISCUSSÁO}

A obtenção dos resultados foram feitas após a análise criteriosa dos questionários respondidos pelos voluntários e por meio delas foi possível traçar as percepções que os pacientes possuem em relação ao processo de adoecimento.

O último censo brasileiro de diálise da Sociedade Brasileira de Nefrologia (SBN) realizado em 2018 aponta que de 48 mil pacientes em diálise em 2002 o número aumentou para mais de 133.000 pacientes em 2018 e que mais de $90 \%$ destes pacientes encontramse em hemodiálise, o que demonstra que ao longo dos anos houve uma redução no uso da diálise peritoneal como um método de terapia renal alternativa à hemodiálise. ${ }^{5}$

Considerando a projeção do censo de 2018 da SBN apenas 7\% (sete por cento) do total de municípios brasileiros têm unidades de diálise, pois que cerca de 108.000 (cento e oito mil) pacientes têm seu tratamento financiado única e exclusivamente pelo SUS, por meio de uma rede de cerca 770 (setecentos e setenta) clínicas credenciadas para este fim, distribuídas em somente 350 (trezentos e cinquenta) municípios. Além disso, a quantidade de vagas disponibilizadas para a população, nos últimos 15 anos não acompanhou o aumento da população em terapia renal substitutiva-TRS. ${ }^{5}$ 
DOI: 10.18606/2318-1419/amazonia.sci.health.v9n2p17-29
RODRIGUES KS, SANTOS, MR. DIAS, AK. COUTO, GBF. PEREIRA, RA. MARKUS, GWS.

Qualidade de vida de pacientes renais crônicos em tratamento dialítico hemodialítico em Guaraí- TO

Gráfico 01: Taxa estimada de pacientes em terapias renais substitutivas no município de Guaraí - TO em outubro de 2020.

\section{Prevalência de Hemodiálise e Diálise Peritoneal na população de Guaraí-TO}

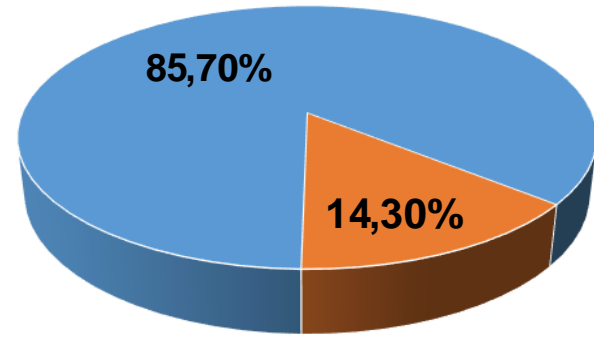

- Hemodiálise - Diálise Peritoneal

Fonte: Os autores, 2020.

Segundo a Sociedade Brasileira de Nefrologia, a Diálise Peritoneal é uma modalidade terapêutica que abrange várias vantagens, como baixo custo para aquisição, manutenção da função renal residual, menos instabilidade hemodinâmica, além de conservar os acessos vasculares e flexibilidade dos horários para realização diária DP. Em contrapartida a Hemodiálise permanece em $1^{\circ}$ lugar no ranking das terapêuticas, sendo de maior alcance dentro das terapias renais. ${ }^{8}$

Os dados obtidos na pesquisa foram estatisticamente relevantes e concordantes no que diz a Sociedade Brasileira de Nefrologia, apresentando um alto índice para hemodiálise com um percentual mais de $80 \%$ dos pacientes pesquisados que realizam as TRS, enquanto somente $14,3 \%$ realizam dialise peritoneal no município de Guaraí - TO. A modalidade de Hemodiálise é usada de forma Intermitente ou Convencional, os pacientes comparecem aos centros de diálise 3 vezes durante a semana sendo conectados à máquina por um período equivalente a 4 horas. Essas idas aos centros de diálise são necessárias, porém são desgastantes para os pacientes, que as vezes precisam percorrer quilômetros por estradas precárias. Outrossim, a colocação da fistula arteriovenosa é um processo doloroso para o paciente e colabora para diminuição da qualidade de vida. ${ }^{6}$

Partindo dessa premissa nota-se uma diferença de custo para realização das terapias substitutivas, onde a dialise peritoneal é totalmente de responsabilidade dos estados, na qual fornece todos os equipamentos e treinamento necessários, já a 
hemodiálise é uma parceria de estados e municípios, tanto nos custos financeiros com transportes e alimentação destinados a esses portadores em tratamento de TRS.

Gráfico 02: Valores estimados do questionário "WHOQOL" de qualidade de vida sobre o domínio relacionado à dor ou desconforto físicos nos pacientes em terapias renais substitutivas

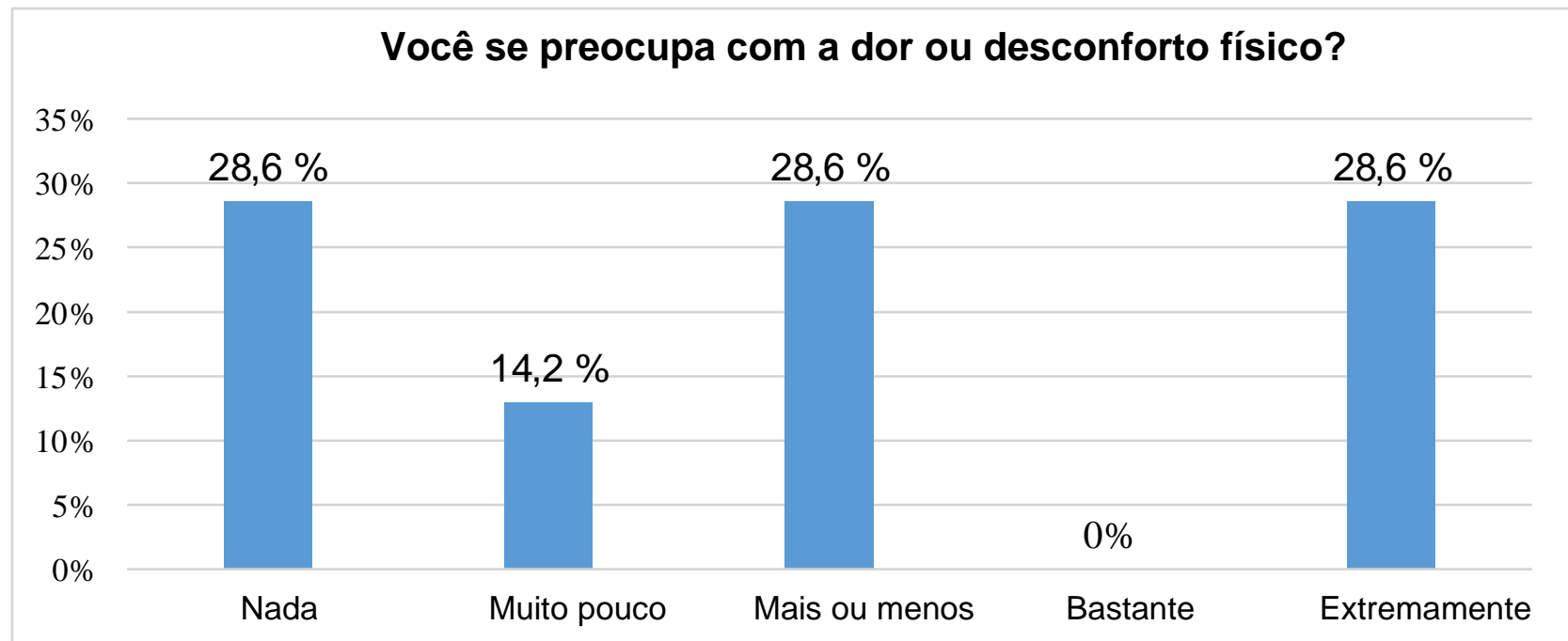

Fonte: Os autores, 2020.

O gráfico nos mostra que em relação ao domínio do questionário referente a dor ou desconforto físico $71,4 \%$ da amostra estudada não se preocupam em nada ou preocupamse muito pouco com as dores advindas das comorbidades ou ocasionadas em consequência do tratamento, em contrapartida, $28,6 \%$ tem extrema preocupação com as dores ou desconforto físico que surgem no dia-a-dia.

Os pacientes renais crônicos passam por uma brusca mudança em seu estilo de vida e convive com limitações em seu cotidiano e sintomas associados às comorbidades como hipotensão, cãibras musculares, cefaleia, dor torácica, dor lombar, como visto anteriormente, ainda que as terapias dialíticas mantenha a vida, as doenças sistêmicas adjacentes e as síndromes dolorosas acometem a grande maioria dos doentes. ${ }^{7}$

Os resultados obtidos não condizem com o autor supracitado, pois as variáveis encontradas evidenciam que o maior índice da amostra não se preocupa com a dor ou desconforto físico ou não a sentem a ponto desta lhe causar inquietações.

Segundo a Sociedade Brasileira para o Estudo da Dor (SBED) a dor é retratada como uma experiência sensitiva e emocional desagradável associada à lesão real ou 
potencial dos tecidos. Considerada pessoal e subjetiva de caráter multidimensional. A dor é ainda definida como um fenômeno que abrange as várias dimensões do ser e engloba fatores físicos, psicológicos, sociais, sendo considerada um problema de magnitude significativa por acometer um índice tão grande de pessoas portadoras por doenças crônicas. ${ }^{9}$

Gráfico 03: Dados referentes à qualidade do sono, utilizando o instrumento de pesquisa "WHOQOL", aplicados aos pacientes com insuficiência renal em terapias substitutivas

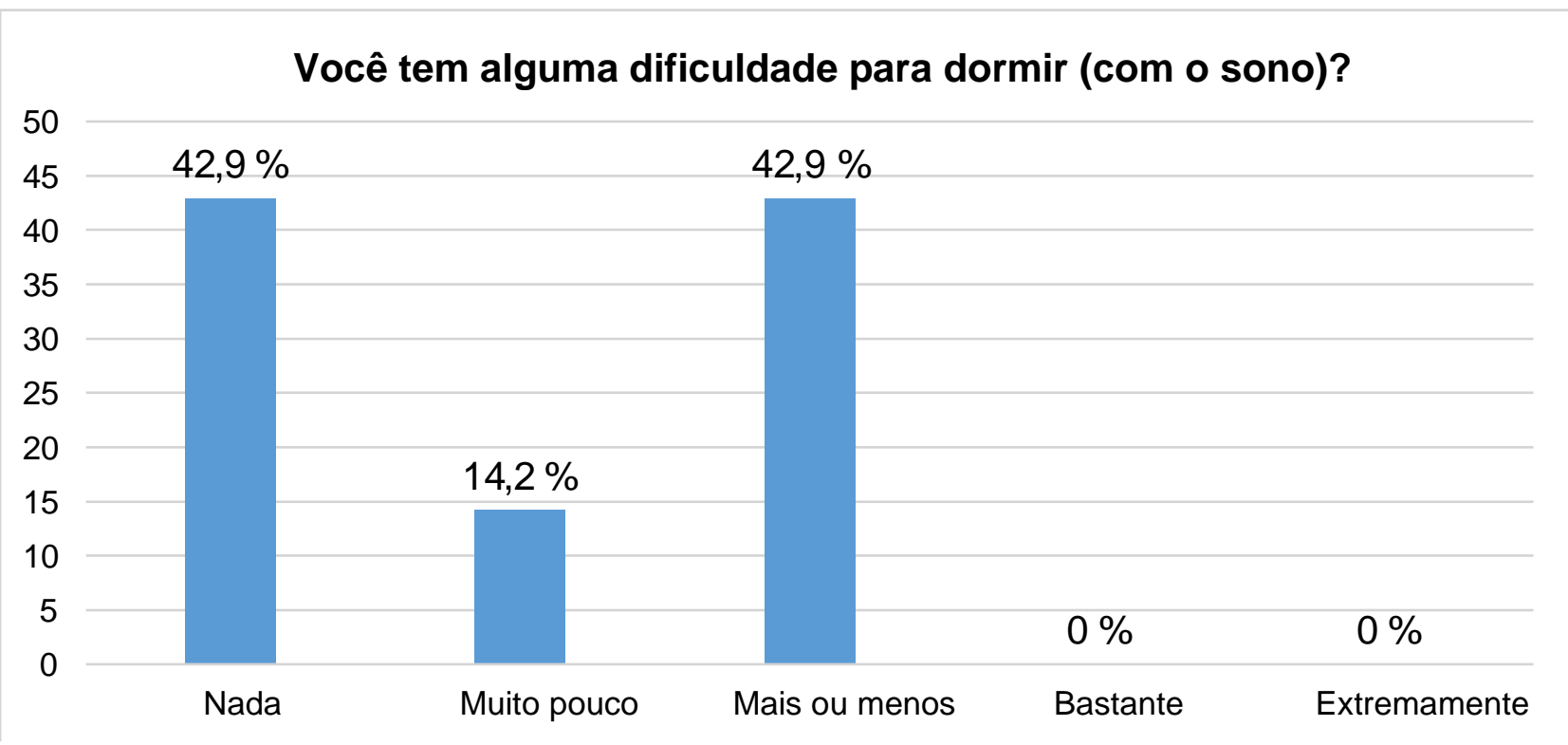

Fonte: Os autores, 2020.

Os dados acima evidenciam que dos participantes da pesquisa, 42,9\% não possuem dificuldades para dormir e relatam que a qualidade do sono encontra-se adequada, outros $42,9 \%$ da amostra classifica como mais ou menos a dificuldade para repousar e 14,2\% apresentam pouca dificuldade em pegar no sono fator este, tranquilizante para os doentes renais crônicos em conseguir manter um padrão de sono satisfatório.

Os resultados obtidos vão de encontro as Teorias das Necessidades Humanas Básicas de Wanda Horta, ratificando a importância de manter o sono e repouso em equilíbrio, de maneira dinâmica, visto que a privação do sono vem acompanhado de consequências que afetam negativamente todas as dimensões da vida humana. ${ }^{10}$

É notável as mudanças que ocorrem em relação ao sono ao longo da vida. Caracteriza-se por um estado de desligamento do organismo do meio de maneira natural 
e reversível ocasionando o descanso do corpo e permitindo alterações das funções cognitivas e sistêmicas. As Terapias Renais Substitutivas e o repouso têm uma ligação complexa que retrata interferência dos fatores fisiológicos e psicológicos que podem propiciar o desenvolvimento dos distúrbios de quietação, acarretando consequências para a saúde e decadência da qualidade de vida. ${ }^{11}$

Gráfico 04: Percentual de pacientes avaliados durante a pesquisa em relação a algum sentimento de tristeza ou depressão presente no dia a dia.

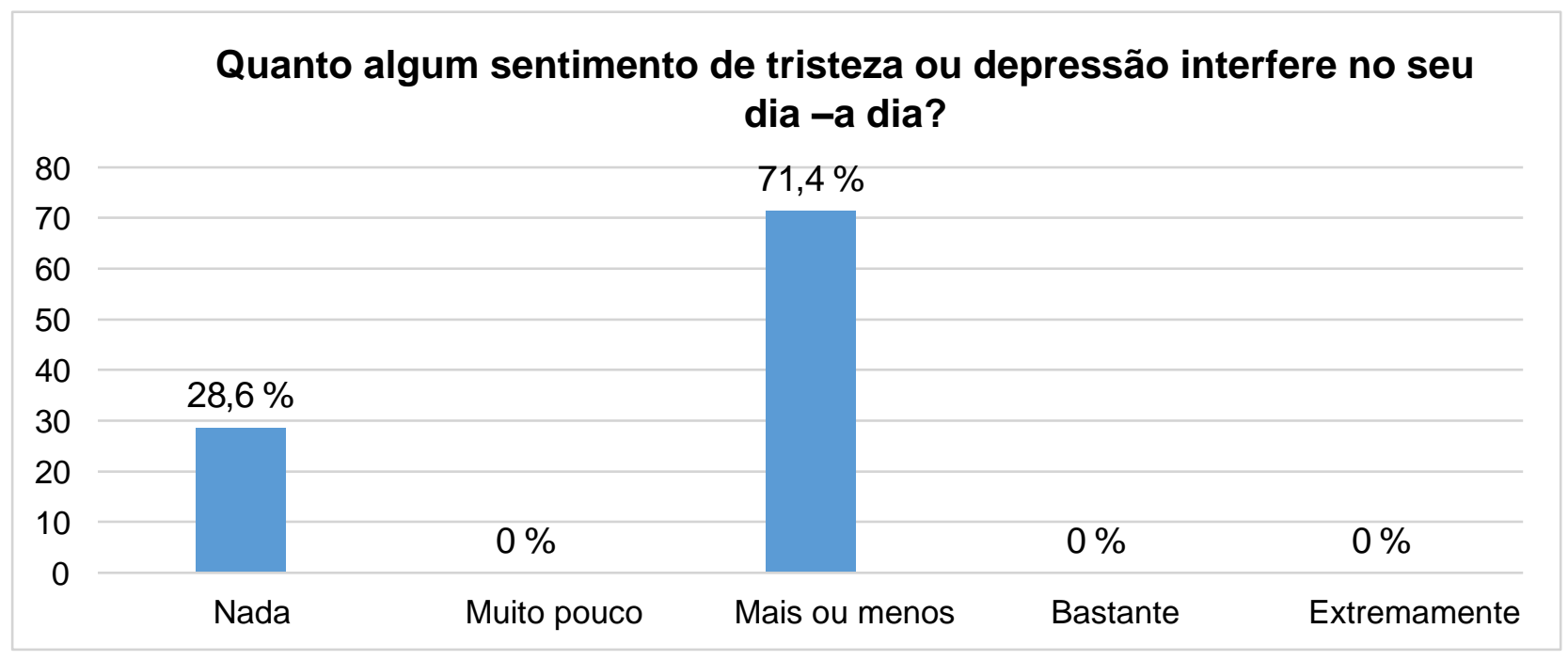

Fonte: Os autores, 2020.

Constata-se no gráfico 4 que dentre os participantes da pesquisa $71,4 \%$ dos entrevistados referem sentir alguma tristeza ou depressão quando lembram de suas limitações em prol do tratamento, a partir desse dado é possível inferir que estes pacientes têm dificuldades em projetar planos à longo prazo por certo medo do futuro, em contrapartida, $28,6 \%$ da amostra estudada relatam não vivenciar sentimento prostração em seu cotidiano.

Partindo desse pressuposto, ao avaliar o parâmetro emergido relacionado à depressão, o estudo foi concordante $\operatorname{com}^{12}$, onde o autor cita o medo e a sensação de inutilidade como fator desencadeante desse distúrbio. Avaliando mais a fundo, quando pensamos na possibilidade de depressão não devemos desvincular o padrão de sono sendo também um fator de risco, pois a maioria das doenças mentais começam com essas alterações. 
Diante disso, no contexto do adoecimento e da necessidade de realização do tratamento que os pacientes vivenciam, percebe-se que estes passam por uma fase de estresse, resistência e exaustão, podendo desencadear não só depressão e ansiedade, como também, o medo, sensação de inutilidade, medo do prognostico, insegurança, baixa autoestima. $^{13}$

Estudos têm demonstrado associação entre depressão e a doença renal crônica. Os pacientes que realizam as terapias substitutivas (hemodiálise e diálise peritoneal) são indivíduos mais suscetíveis ao sentimento de tristeza e estresse por vivenciarem diariamente condições particulares limitantes, como controle rigoroso da ingesta hídrica, calórica e restringimento da vida laboral. ${ }^{13}$

Gráfico 05: Percentual de doentes renais crônicos avaliados pelo questionário "WHOQOL" referente ao domínio qualidade de vida.

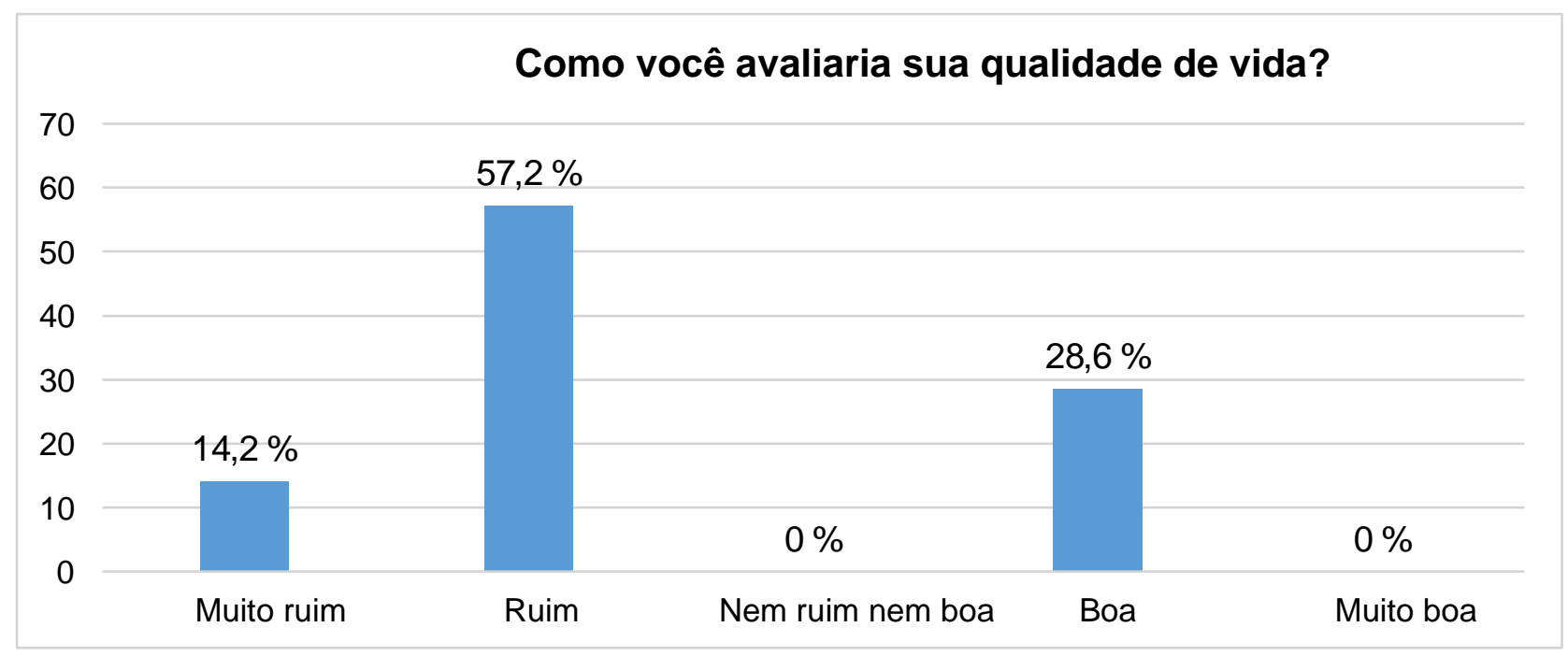

Fonte: Os autores, 2020.

A figura acima evidencia que em relação ao domínio QV da população estudada, $71,4 \%$ a classifica como ruim ou muito ruim, o que notadamente presume que a doença e as terapias indicadas afetam significativamente a vida do indivíduo e apenas $28,6 \%$ relatam ter uma boa QV mesmo com o estresse e mal-estar que as TRS provocam rotineiramente, estes entendem a importância do tratamento para a manutenção da vida.

Partindo dessa premissa, no que concerne os parâmetros de avaliação da qualidade de vida, este estudo é concordante ao que diz o autor ${ }^{14}$, relata que as dificuldades das pessoas acometidas pela insuficiência renal podem interferir em todos os aspectos da vida, 
tanto social, físico, cultural, entretanto, o apoio da família e da equipe multiprofissional envolvida nos cuidados e na recuperação fazem toda a diferença para que esses pacientes enfrentem as limitações dos tratamentos impostos e melhore progressivamente a qualidade de vida.

A qualidade de vida é definida como a percepção do indivíduo em relação a sua vida, no contexto cultural e de valores intrínsecos relacionados a seus objetivos, expectativas, crenças, abrangendo as dimensões física, psicológica, sociais e meio ambiente, em síntese, a avaliação da qualidade de vida tornou-se um bom indicador de saúde dos indivíduos ${ }^{15}$.

A qualidade de vida não só dos portadores de doenças crônicas como também de toda população, vem gerando grandes repercussões nas políticas públicas quanto a importância nos cuidados e promoção do bem-estar biopsicossocial. Tal fato é de extrema importância porque interfere diretamente na percepção que os indivíduos possuem sobre si, saúde/doença, aspectos estes que refletem na vida do ser humano ${ }^{16}$.

Gráfico 06: Dados relacionados à vida sexual das pessoas acometidas por insuficiência renal crônica, utilizando como instrumento de pesquisa o questionário validado "WHOQOL".

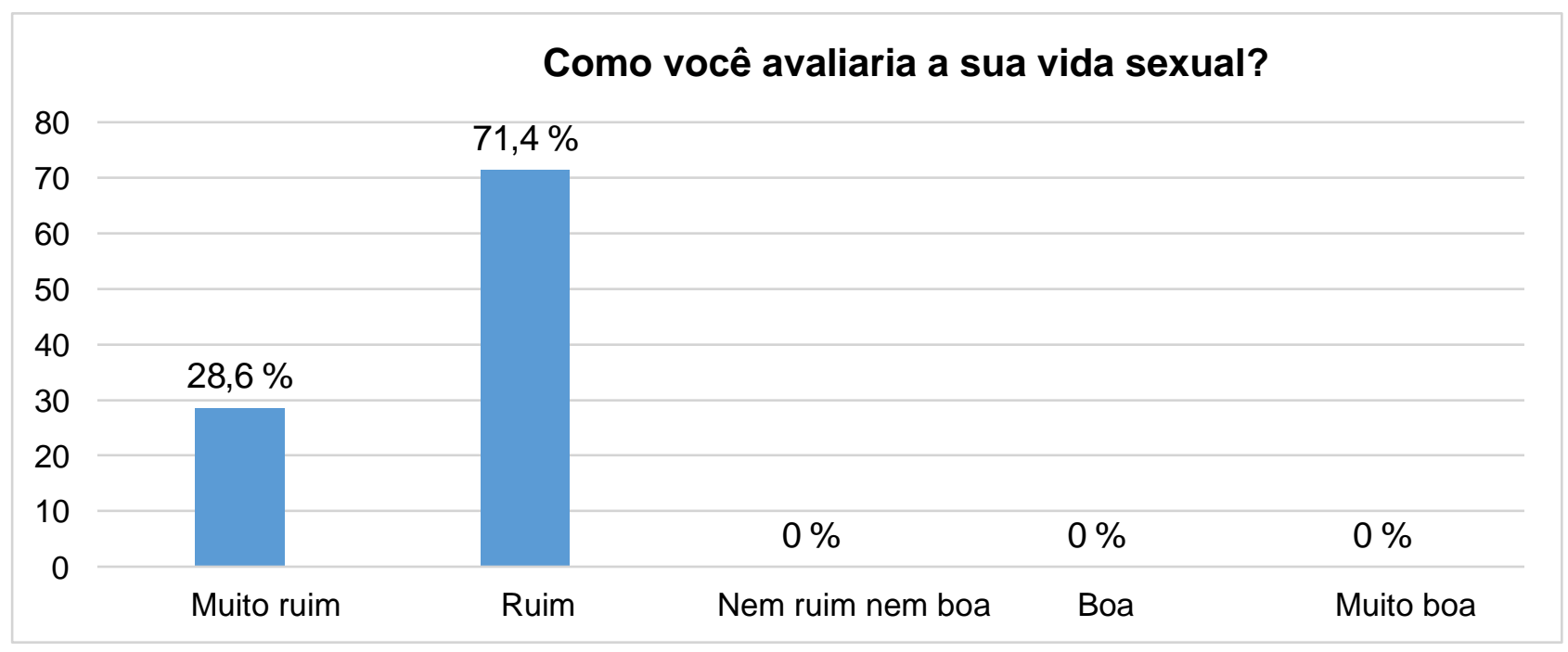

Fonte: Os autores, 2020.

Os dados encontrados na figura 6 mostra que em relação a qualidade da vida sexual, $71,4 \%$ a avalia como ruim e $28,6 \%$ a classificam como sendo muito ruim. Esse dado deixa claro que a insuficiência renal crônica acomete de forma severa a vida dessa população, com o aumento de distúrbios orgânicos e psicológicos que ocasionam a 
disfunção sexual inerente às terapias indicadas, além do fato de que muitos medicamentos usados no tratamento da doença também podem afetar a funcionalidade sexual e assim contribuir com o declínio da qualidade de vida dessa população.

Diante do exposto, os resultados obtidos condizem com o estudo do autor ${ }^{17}$ observou que a disfunção sexual é mais um agravante na insuficiência renal e acarreta, além das limitações físicas e sociais, o que consequentemente ocasiona a piora da qualidade de vida desse grupo de pessoas.

A saúde sexual relaciona-se ao aspecto somático, emocional, intelectual e social do indivíduo e o comprometimento de algum desses fatores repercutem de maneira negativa na função sexual, logo, afeta diretamente a qualidade de vida das pessoas, sendo caracterizado como um problema multidimensional que gera frustação, dor e dificuldades para interação social. ${ }^{17}$

A insuficiência renal acomete várias funções no organismo do indivíduo, comprometendo a qualidade de vida de forma restrita em vários âmbitos, inclusive na vida sexual, visto que os doentes renais crônicos se sentem desanimados e menos atraentes sexualmente, e passam postergar as práticas sexuais. É sabido que essa doença expressa dificuldades para ereção, além da insatisfação com o próprio corpo, fatos estes que exigem apoio multidisciplinar de profissionais da saúde, como psicólogo, enfermagem e família, levando em conta que essas adaptações são fundamentais e necessárias para satisfação fisiológica e bem estar de cada indivíduo. ${ }^{18}$

\section{CONSIDERAÇÕES FINAIS}

Constatou-se nesse estudo que a qualidade de vida dos pacientes renais crônicos apresenta-se de forma negativa em função da sobrecarga causada pela doença e das limitações frente às rotinas exigidas pelos tratamentos em DP e HD, além disso, foi possível estimar a expectativa em relação aos aspectos biopsicossociais.

A pesquisa demonstrou ainda que há maior número de paciente em hemodiálise se comparados aos pacientes que realizam a diálise peritoneal. É sabido que a hemodiálise tem um custo mais elevado para os cofres públicos, pois necessita de clínicas especializadas, transporte, alimentação e equipe multiprofissional à disposição. Enquanto que para a realização da Diálise peritoneal, o custo é mais acessível para o Sistema Único de Saúde (SUS), pois é feita no próprio domicílio, sob auxilio da família, tendo a disposição um local adaptado para a modalidade que o tratamento exige. 
Outrossim, esta pesquisa não tem a pretensão de enaltecer uma terapia em detrimento da outra, visto que as duas modalidades citadas são essenciais para continuidade da vida dos pacientes e cada uma delas tem suas vantagens e desvantagens.

Considerando a pouca publicação de estudos sobre a magnitude da doença no Tocantins e, em Guaraí/TO, sugere-se que a doença apesar de aumentar cada vez mais no Brasil, ainda é negligenciada sob o ponto de vista de prevenção e promoção, e com diagnóstico tardio, fato que justifica a importância desse estudo.

Diante do exposto, reforça-se a necessidade de medidas que possam contribuir para ações de prevenção, diagnóstico, tratamento precoce, acompanhamento multiprofissional com vistas a tratar as comorbidades e possíveis intercorrências associadas ao novo estilo de vida, a fim de que se reduza a morbimortalidade e as incapacidades físicas, mentais e psicológicas em decorrência da doença.

\section{REFERÉNCIAS}

1. Rocha IA, et al. O Custo do Atendimento aos Pacientes com Doença Renal Crônica (DRC), em Fase Não Dialítica de um Hospital Universitário. J.res. fundam. care. Online, 2018. DOI: 10.9789/2175-5361.2018.v10i3.647-655

2. Pinho NA, Silva GV, Pierin AMG. Prevalence and factors associated with chronic kidney disease among hospitalized patients in a university hospital in the city of São Paulo. SP, Brazil. J Bras Nefrol. 2015. DOI: https://doi.org/10.5935/0101-2800.20150013

3. Sesso C. et al. Inquérito Brasileiro de Diálise Crônica 2016. J Bras Nefrol 2017.Disponível em: https://doi.org/10.5935/0101-2800.20170049

4. Rebouças CBA, Araújo MM, Braga FC, Fernandes GT, Costa SC. Avaliação da qualidade de vida de deficientes visuais. Rev Bras Enferm 2016. https://doi.org/10.1590/2175-8239jbn-2018-0152

5. Federação Nacional de Associações de Pacientes Renais e Transplantados do Brasil FENAPAR. SOCIEDADE BRASILEIRA DE NEFROLOGIA (SBN), Associação Brasileira dos Centros de Diálise (ABCDT), e Federação Nacional de Associações de Pacientes Renais e Transplantados do Brasil (FENAPAR, 2020). Acesso em: 21de outubro de 2020

6. Gonzalez-Bedat M, Rosa-Diez G, Pecoits-Filho R, Ferreiro A, García-García G, Cusumano A, et al. Burden of disease: prevalence and incidence of ESRD in Latin America. Clin Nephrol.

2015. DOI: $10.5414 / \mathrm{cnp} 83 \mathrm{~s} 003$

7. Martinez JE, Grassi DC, Marques LG. Análise da aplicabilidade de três instrumentos de avaliação de dor em distintas unidades de atendimento: ambulatório, enfermaria e urgência. Rev. Bras. Reumatol. São Paulo, 2011. Disponível em: https://doi.org/10.1590/S048250042011000400002 . 
8. Sociedade Brasileira de Nefrologia (Brasil). Doenças comuns.

Tratamento. São Paulo, 2017. Disponível em: http://dx.doi.org/10.1590/2175-8239jun2018-0178

9. Siqueira JTT, Annes AH. Sociedade Brasileira para o Estudo da Dor (SBED), 2014. Disponível em:

https://sbed.org.br/wpcontent/uploads/2019/01/01 quandoadorsetorna.pdf.

10. Horta WA. Processo de enfermagem. Rio de Janeiro: Guanabara Koogan; 2011. Disponível em: https://www.scielo.br/pdf/tce/v20n3/15.pdf.

11. Karatas A, Canakci E, Turkmen E. Comparison of Sleep Quality and Quality of Life Indexes with Sociodemographic Characteristics in Patients with Chronic Kidney Disease. Nigerian Journal Clinical Pratice. 2018.

DOI: $10.4103 /$ njcp.njcp 14618

12. Santos, ACM, Nakasu, MVP. Prevalência de sintomas de estresse e depressão em pacientes renais crônicos submetidos à hemodiálise em um hospital escola do sul de Minas Gerais. Revista de Ciências em Saúde. v. 7, n.2, p. 16-22. 2017. DOI: https://doi.org/10.21876/rcsfmit.v7i2.659

13. Lima AB. Estresse, depressão e suporte familiar em pacientes em diálise peritoneal e hemodiálise, 2016. Disponível em: http://hdl.handle.net/11449/138927

14. Kirchner RM, et al. Análise do estilo de vida de renais crônicos em hemodiálise. 0 Mundo da $\quad 2019 . \quad$ Saúde. Disponível em: http://www.saocamilosp.br/pdf/mundo saude/88/07 AnalisedoEstilodevida.pdf

15. THE WORLD HEALTH ORGANIZATION QUALITY OF LIFE ASSESSMENT (WHOQOL): position paper from the World Health Organization. Soc Sci Med 1995; 41:1403-9. PMID: 8560308 DOI: 10.1016/0277-9536(95)00112-k

16. Machado LRC, Car MR. A Dialética da Vida Cotidiana de Doentes com Insuficiência Renal Crônica: entre o inevitável e o casual. Rev. Esc. Enfermagem USP, 2003. Disponível em: https://doi.org/10.1590/S0080-62342003000300004.

17. Prado DS, Mota VP, Lima TI. Prevalence of sexual dysfunction in two women groups of different socioeconomic status. Rev Bras Ginecol Obstet. 2019. Disponível em: https://doi.org/10.1590/S0100-72032010000300007

18. Rodrigues DF, Scwartz E, Santana MG, Zilmer JBV, Viegas AC, Santos BP, et al. Experiencias de los hombres sometidos a hemodiálisis acerca de su sexualidade. Av Enferm. 2015. Disponível em:

http://www.scielo.org.co/pdf/aven/v29n2/v2 9n2a05.pdf,

19. Marinho AW. et al. Prevalência de doença renal crônica em adultos no Brasil: revisão sistemática da literatura. Cad. Saúde Colet., Rio de Janeiro, 2017. DOI: https://doi.org/10.1590/1414-462x201700030134 